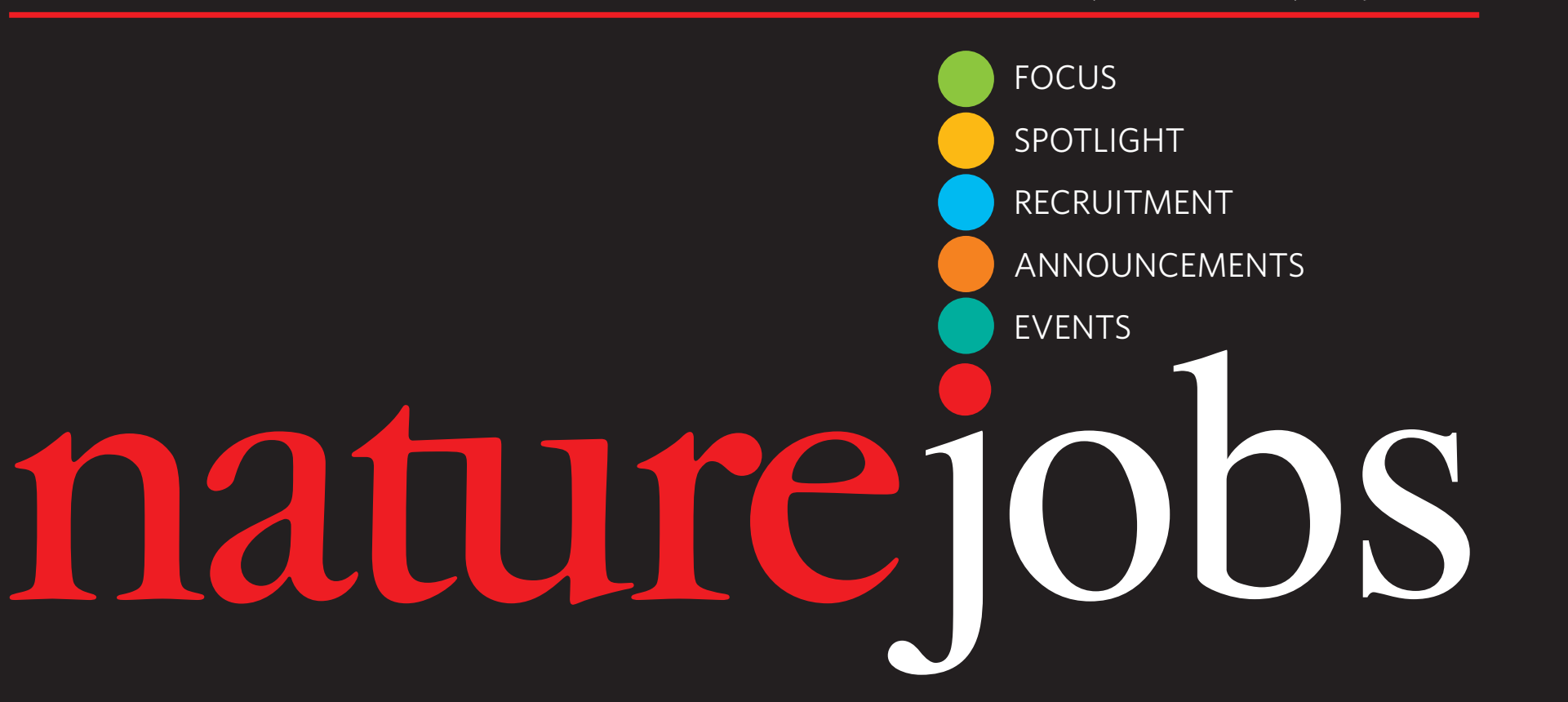

\title{
A howl of hope
}

This year is the 50th anniversary of the landmark poem Howl by Allen Ginsberg, whose opening line is "I saw the best minds of my generation destroyed by madness." So it's timely to see how the verse (not including the stuff about sex and drugs, which is, admittedly, about $75 \%$ of it) might apply to the next generation of scientists.

In the first part of Howl, Ginsberg writes about a generation who "passed through universities with radiant cool eyes". So far, so good. One can interpret this as guarded optimism in the quest for knowledge, perhaps exemplified by today's student pipeline, which is producing an ample number of scientists.

A phrase halfway through the middle section, "ashcans and unobtainable dollars", is a bit foreboding, especially if you consider the flattening of the US National Institutes of Health research budget to be equivalent to unobtainable dollars that could put young researchers' careers into the ashcan.

The next section is even more ominous, as it rages against the machine of "Moloch", a god who requires human sacrifice. In science, this could include a study

CONTACTS

Publisher: Ben Crowe

Editor: Paul Smaglik

Assistant Editor: Corie Lok

US Head Office, New York

75 Varick Street, 9th Floor,

New York, NY 10013-1917

Tel: +1 8009897718

Fax: +18009897103

e-mail: naturejobs@natureny.com

US Sales Manager/Corporations: Peter Bless

Classified Sales Representatives

Tel: +18009897718
New York/Pennsylvania/

Latin America: Andrew Bennie

Midwest USA/Maryland/

NIH: Wade Tucker

East USA/Canada:

Janine Taormina

San Francisco Office

Classified Sales Representative:

Michaela Bjorkman

West USA/West Corp. Canada

225 Bush Street, Suite 1453

San Francisco, CA 94104

Tel: +14157813803

Fax: +14157813805

e-mail:m.bjorkman@naturesf.com

section, an institutional review board or a regulatory agency, all of which have the power to halt a research project as they see fit.

But here, too, there's hope. Scientists have built up ways to appease, if not beat, their individual Molochs by banding together under student and postdoc organizations, professional societies, lobbying efforts and mentoring schemes.

Howl chronicles how an individual can be crushed by an institution. It could be read as a warning for scientists to band together, avoid alienation and triumph over obstacles. Perhaps scientists who avoid isolation could rewrite the cautiously triumphant ending as follows:

"Holy the supernatural extra brilliant intelligent funding of the grant."

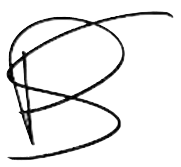

\section{Paul Smaglik, Naturejobs editor}

European Head Office, London

The Macmillan Building,

4 Crinan Street,

London N1 9XW, UK

Tel: +44 (0) 2078434961

Fax: +44 (0) 2078434996

e-mail:naturejobs@nature.com

European Sales Manager:

Andy Douglas (4975)

Advertising Production Manager: Billie Franklin

To send materials use London address above.

Tel: +44 (0) 2078434814

Fax: +44 (0) 2078434996

e-mail: naturejobs@nature.com
Naturejobs web development: Tom Hancock Naturejobs online production: Niamh Shields

European Satellite Office

Patrick Phelan

e-mail:p.phelan@nature.com

Japan Head Office, Tokyo

Chiyoda Building,

2-37 Ichigayatamachi,

Shinjuku-ku,

Tokyo 162-0843

Tel: +81332678751

Fax: +81332678746

Asia-Pacific Sales Director: Rinoko Asami

e-mail: r.asami@naturejpn.com 